A further clinical objective evaluation of children with acute head injury includes tests of neuropsychological function. Investigators from the Dept Pediatrics, Univ of Maryland, Baltimore, report cognitive deficits immediately after closed head injury in adolescents that may interfere with school, home and peer activities ( Oct $1988 ; \underline{142}: 1048$ ).

\title{
LEARNING AND BEHAVIOR
}

\section{SUCROSE, MOTOR ACTIVITY, AND LEARNING}

The effects of sugar (sucrose) on the behavior of 30 preschool children ( 20 boys and 10 girls, mean age 5 years 4 mos) and 15 elementary school children ( 6 boys and 9 girls, mean age 7 yrs 2 mos) were investigated by psychologists from Colorado State University, Fort Collins, CO, and the Univ of Mississippi Med Cntr, Jackson, MS. Parents and teachers questioned before the study complained that the child was behaviorally sensitive to sugar in approx 508 of subjects. Two preschool children had been considered hyperactive by the school director. A basic breakfast included a $4 \mathrm{oz}$ orange flavored drink of high sucrose content (50g), low-sugar (6.25g) or aspartame (122 mg), randomly selected, 5 days on each, using a double-blind control design. The mean sucrose intakes for the high, low, and "control" aspartame conditions were 2.26, 0.28, and $0.00 \mathrm{~g} / \mathrm{kg}$, respectively, and the total carbohydrate contents of breakfast averaged $3.95,1.88$, and $1.54 \mathrm{~g} / \mathrm{kg}$, respectively.

On cognitive measures, girls made significantly more errors on a paired-associate learning task performed 20-30 min following a high-sugar content breakfast when compared to a low-sugar meal, whereas boys were unaffected. On global ratings, younger preschool children were affected differently than older children. On an Abbreviated Conners Teacher Rating Scale completed before lunch, both boys and girls were more active in behavior after the high sugar meal than that of low sugar content. Measures of behavior by observation for fidgetiness, change in activity, running, vocalization and aggressiveness and other cognitive measures involving matching and academic tasks failed to demonstrate changes after sugar ingestion. (Rosen LA et al. Effects of sugar (sucrose) on children's behavior. $\mathrm{J}$ Consulting Clin Psychol 1988; $56(4): 583-589$ ).

COMMENT. Evidently, the effects of sugar on children's behavior is not yet resolved. This study demonstrates significant adverse effects although the authors conclude that these are minimal in degree. Certain limitations of the study design are admitted: 1. The sugar challenge dose was the same for all subjects and younger and smaller children, affected differently, received larger amounts 
than did older and larger children. The design was not adequate to pinpoint the amount of sugar that may cause deleterious effects. 2. The prior dietary history of the subjects was unknown, and those accustomed to consuming large amounts of sugar may have reacted differently from children who usually ate low sugar meals. 3. The assumption that aspartame used as a control is innocuous may not be correct (see ped Neur Briefs Nov 1987;1:45). Further work on possible behavioral effects of sucrose is clearly indicated. The only proven contraindication to excess sugar in a child's diet is that emphasized by the dental profession.

\section{LANGUAGE DEVELOPMENT AND OTITIS MEDIA}

The effects of otitis media on early language development assessed at 1 year of age in 46 high-risk and low birthweight infants and 19 healthy full-term babies were examined in the R.F. Kennedy Center's Clinical Research Center for Communicative Disorders, Albert Einstein College of Medicine, Bronx, N.Y. Patients were largely of Hispanic background but subjects were recruited from English speaking families. By pneumatic otoscopy examination, 15 were otitis free and 12 were bilaterally otitis positive. The Bayley Scales of Infant Development and the Sequenced Inventory of Communication Development (SICD) Receptive scale showed no significant differences in the 2 groups, but the SICD expressive language scores were significantly lower in the otitis positive group. Full-term infants with frequent episodes of bilateral otitis media performed no better than high risk infants with otitis media (Wallace IF et al. Otitis media and language development at 1 year of age. $\mathrm{J}$ Speech and Hearing Disorders Aug $1988 ; \underline{53}: 245-251$ ).

COMMENT. Infants who suffer repeated episodes of bilateral otitis media during the first year of life are at risk for expressive language difficulties. These findings are important in the evaluation of infants who have experienced perinatal insults such as asphyxia and are late in acquiring expressive language. The delay may be caused by peripheral factors as much as damage to cerebral language centers.

Disorders of higher cerebral function including developmental language are reviewed by $\mathrm{Dr}$. Isabelle Rapin at the Albert Einstein College of Medicine, Bronx, N.Y. (AJDC Oct1988; 142:1119-1124). Included under the differential diagnosis of language delay are the following: hearing loss, mental deficiency, dysphasia, autism, mutism, dysarthria and structural respiratory tract abnormalities. No meaningful words by age 18 months or no meaningful phrases by age 24 months should be cause for concern and the use of language intervention programs. 